\title{
MORFOMETRIA DO ENCÉFALO E FORMAÇÃO DO CIRCUITO ARTERIAL EM EQUINOS MESTIÇOS
}

\author{
SARA OtOni da Silva Moraes ${ }^{1}$, MARCElo SAlvador Gomes ${ }^{2}$, PAUlo Oldemar Scherer $^{3}$, \\ MARCELO ABIDU FIGUEIREDO ${ }^{3}$

\footnotetext{
${ }^{1}$ Graduanda da Universidade Federal Rural do Rio de Janeiro, Rio de Janeiro, RJ, Brasil.

${ }^{2}$ Pós-Graduando da Universidade Federal Rural do Rio de Janeiro, Rio de Janeiro, RJ, Brasil.

${ }^{3}$ Professores Doutores da Universidade Federal Rural do Rio de Janeiro, Rio de Janeiro, RJ, Brasil marceloabidu@gmail.com
}

\begin{abstract}
O objetivo desta investigação foi caracterizar a morfometria do encéfalo e a formação do circuito arterial em equinos adultos mestiços de ambos os sexos. A fixação foi feita através de cânula plástica introduzida na artéria carótida comum com solução de formaldeído a $10 \%$. Em seguida, foram feitas repleções vasculares com solução aquosa de Petrolátex S65 corado com pigmento Suvinil vermelho. Procedeu-se a craniotomia, remoção e morfometria dos encéfalos e dissecção para a observação das ramificações principais da artéria basilar e formação do circuito arterial. A média mais erro padrão da média do
\end{abstract}

comprimento da artéria basilar foi de $3,629 \mathrm{~cm} \pm 0,1550$ nos machos e 4,423 $\mathrm{cm} \pm 0,1990$ nas fêmeas ( $\mathrm{p}=0,0037$ ). Em todos os equinos dissecados, a artéria basilar originouse da convergência das artérias vertebrais, emitindo a artéria cerebelar caudal, ramos variados para bulbo e ponte, ramos terminais e artérias cerebelares rostrais. As artérias da base do encéfalo estiveram na dependência dos sistemas carótico e vértebrobasilar. O circuito arterial do cérebro apresentou-se fechado rostral e caudalmente em $100 \%$ dos animais dissecados.

PALAVRAS-CHAVE: cérebro, circuito arterial, equino

\section{MORPHOMETRY OF THE BRAIN AND ARTERIAL CIRCUIT FORMATION IN CROSSBREAD HORSE}

\section{ABSTRACT}

The aim of this study was to describe the main branches of the basilar artery, formation of the arterial circuit and the morphometry of the brain in adult crossbreed horses of both sexes. The setting was made by plastic tube inserted into the common carotid artery with $10 \%$ formaldehyde solution. After fixed, latex solution stained with pigment was injected. Craniotomy, morphometry of the brain and dissection were made to identify the main branches of the basilar arteries and the formation of the arterial circuit. The mean and standard error of the mean of the basilar

KEYWORDS: arterial circuit, brain, horse.

\section{INTRODUÇÃO}

As doenças que acometem o sistema artery was $3.629 \mathrm{~cm} \pm 0.1550$ in males and $4.423 \mathrm{~cm} \pm$ 0.1990 in females $(p=0.0037)$. In all animals the basilar artery originated from the fusion of the vertebral arteries giving rise to caudal cerebellar arteries, different numbers of branches to bulbus and pons, terminal branches and rostral cerebellar arteries. The arteries of the base of the brain depended on the carotid and vertebral-basilar systems. The brain circuit was closed rostrally and caudally in all horses.

nervoso central (SNC) de equídeos representam uma parcela importante das afecções nessas espécies. $\mathrm{O}$ estudo destas e de outras enfermidades 
nas diferentes regiões do país é necessário para estabelecer formas eficientes de controle e profilaxia ${ }^{1}$.

Nos últimos anos tem-se observado grande avanço nas técnicas de diagnóstico por imagem, que se destinam à investigação das estruturas do sistema nervoso central facilitando, dessa forma, o tratamento de diferentes patologias ${ }^{2}{ }^{3}$. Acompanhando o mesmo crescimento, muitas doenças do sistema nervoso também passaram a ser diagnosticadas com mais frequência e precisão, possibilitando muitos avanços na pesquisa biomédica gerando tratamentos cada vez mais seguros e minimamente invasivos ${ }^{4,5}$.

As artérias encefálicas representam a principal fonte de suprimento sanguíneo para o cérebro sendo essenciais na homeostase cerebral. Pela sua importância funcional, vários estudos morfológicos foram realizados em diferentes espécies. No estudo da anatomia regional do sistema nervoso central, os vasos sanguíneos apresentam grande variabilidade numérica, na origem, trajeto e distribuição. Diferentes estudos sobre o ser humano relataram duplicidade ou agenesia das artérias da base do encéfalo ${ }^{6-9}$; entretanto, relatos dessa natureza são escassos na anatomia veterinária aplicada, dificultando muitas vezes os estudos angiográficos.

O conhecimento preciso das variações vasculares possui grande importância em um programa de sistematização da prática de diagnóstico por imagem e também da prática cirúrgica em animais domésticos. Variações na anatomia vascular cerebral podem aumentar a morbidade nos procedimentos cirúrgicos e intervencionistas ${ }^{10}$. A irrigação sanguínea para o encéfalo apresenta uma considerável variação entre as espécies animais. Tais modificações estão relacionadas com as fontes de suprimento sanguíneo e com a multiplicidade de arranjos das artérias que se distribuem na região do sistema nervoso central. Dados sobre essas fontes de suprimento revelaram-se contraditórios e escassos ${ }^{11}$.

O presente trabalho tem como objetivo caracterizar a morfometria do encéfalo e da formação do circuito arterial em equinos mestiços adultos de ambos os sexos.

\section{MATERIAL E MÉTODOS}

Foram utilizadas 30 cabeças de equinos mestiços provenientes do acervo da área de anatomia animal da Universidade Federal Rural do Rio de Janeiro, as quais já haviam sido utilizadas em outros experimentos. Essas cabeças foram previamente identificadas e, através de uma cânula plástica introduzida na artéria carótida comum, fixadas com solução de formaldeído a $10 \%$, preenchendo-se o sistema arterial com solução de petrolátex S65 (Refinaria Duque de Caxias / Petrobrás, Duque de Caxias, Rio de Janeiro, Brasil) corado com pigmento Suvinil vermelho. Posteriormente, as calotas cranianas foram retiradas e incisões foram feitas nas meninges, mergulhando-se em seguida todo o material em solução de formol a $30 \%$, por cinco dias, para complementação da fixação dos encéfalos e coagulação do látex. Para abertura dos crânios, remoção dos encéfalos e dissecação dos vasos da base, foram utilizados instrumentais cirúrgicos de rotina e fórceps odontológico.

Todas as mensurações foram realizadas pelo mesmo observador, utilizando um paquímetro digital da marca Eda. O comprimento dos hemisférios cerebrais foi obtido através da distância entre os pólos frontal e occipital de cada hemisfério, a largura entre a distância do sulco mediano dorsal até o pólo temporal respectivo e a altura compreendida entre a base do lobo piriforme e a superfície dorsal do respectivo hemisfério. Também foram feitas medidas do comprimento e largura do cerebelo.

Os vasos da base do encéfalo foram dissecados e fotografados (Figura 1) para documentação e denominados de acordo com a nomenclatura anatômica veterinária ${ }^{12}$ e com o International Committee on Veterinary Gross Anatomical Nomenclature ${ }^{13}$.

Foram obtidas a média e o erro padrão da média das mensurações do encéfalo e da artéria basilar em ambos os sexos e comparadas através do Teste $t$ não pareado, considerando-se $p<0,05$ significativo. Toda a análise estatística foi realizada no Software Graphpad Prism 5.

\section{RESULTADOS}

A artéria basilar formou-se a partir da convergência da artéria vertebral direita e esquerda em $100 \%$ dos animais. Apresentou-se reta em 11 $(73 \%)$ machos e seis $(40 \%)$ fêmeas, sinuosa em quatro $(27 \%)$ machos e nove $(60 \%)$ fêmeas, sendo única em ambos os sexos. 
Tabela1: Média e erro padrão da média das medidas em centímetros do encéfalo e da artéria basilar em equinos mestiços de ambos os sexos

\begin{tabular}{lcc}
\hline & \multicolumn{2}{c}{ Equinos } \\
\cline { 2 - 3 } & Machos & Fêmeas \\
Comprimento do Hemisfério Direito & $9,165 \pm 0,3814$ & $9,269 \pm 0,2015(\mathrm{p}=0,8105)$ \\
Comprimento do Hemisfério Esquerdo & $9,187 \pm 0,3227$ & $9,408 \pm 0,2034(\mathrm{p}=0,5662)$ \\
Altura do Hemisfério Direito & $6,033 \pm 0,1817$ & $5,889 \pm 0,1506(\mathrm{p}=0,5488)$ \\
Altura do Hemisfério Esquerdo & $5,869 \pm 0,1863$ & $5,803 \pm 0,1276(\mathrm{p}=0,7734)$ \\
Largura do Hemisfério Direito & $3,768 \pm 0,2009$ & $4,345 \pm 0,1370(\mathrm{p}=0,0243)$ \\
Largura do Hemisfério Esquerdo & $3,856 \pm 0,1970$ & $4,343 \pm 0,07935(\mathrm{p}=0,0290)$ \\
Comprimento do Cerebelo & $4,255 \pm 0,1541$ & $4,691 \pm 0,1201(\mathrm{p}=0,0332)$ \\
Largura do Cerebelo & $5,091 \pm 0,1854$ & $5,541 \pm 0,1321(\mathrm{p}=0,0570)$ \\
\hline Comprimento da a. basilar & $3,629 \pm 0,1550$ & $4,423 \pm 0,1990(\mathrm{p}=0,0037)$ \\
\hline
\end{tabular}

A artéria basilar formou-se a partir da convergência da artéria vertebral direita e esquerda em $100 \%$ dos animais. Apresentou-se reta em 11 $(73 \%)$ machos e seis $(40 \%)$ fêmeas, sinuosa em quatro $(27 \%)$ machos e nove $(60 \%)$ fêmeas, sendo única em ambos os sexos.

A artéria basilar emitiu como primeiro ramo a artéria cerebelar caudal única em ambos os antímeros e em ambos os sexos. A artéria cerebelar caudal apresentou simetria de posição em nove $(60 \%)$ machos e quatro $(27 \%)$ fêmeas e, consequentemente, assimetria em seis $(40 \%)$ machos e $11(75 \%)$ fêmeas. Em 100\% dos machos, originouse da artéria basilar em ambos os antímeros. Nas fêmeas, porém, a origem foi variável: em nove $(60 \%)$ fêmeas, a origem foi na artéria basilar em ambos os antímeros; em duas (12\%) fêmeas, esta artéria originou-se do ponto de convergência das artérias vertebrais para formação da artéria basilar, no antímero esquerdo, e diretamente da artéria basilar, no antímero direito; em uma $(6 \%)$ fêmea, no antímero direito, originou-se no da artéria vertebral e no esquerdo na artéria basilar. Em outra (6\%) fêmea, no antímero direito, a artéria originou-se do ponto de convergência das artérias vertebrais para formação da artéria basilar e, no antímero esquerdo, da artéria vertebral; na última fêmea (6\%), a artéria originou-se do ponto de convergência das artérias vertebrais para formação da artéria basilar, em ambos os antímeros.

Em seguida, a artéria basilar emitiu ramos variáveis para ponte e bulbo em ambos os antímeros. Nos machos, a média mais erro padrão da média desses ramos para o antímero direito foi de $4,400 \pm$ 0,1633 e para o esquerdo de 4,200 $\pm 0,1447$ $(\mathrm{p}=0,3672)$. Nas fêmeas, a média mais erro padrão da média dos mesmos ramos para o antímero direito foi de 5,200 $\pm 0,2795$ e para o esquerdo de 5,000 \pm $0,1952(\mathrm{p}=0,5621)$. Comparando o número de ramos entre os sexos, observou-se que, tanto no antímero direito $(p=0,0198)$ quanto no esquerdo $(p=0,0054)$, as fêmeas apresentaram maior número de ramos para ponte e bulbo.

No ponto de bifurcação, a artéria basilar, em sua porção rostral, emitiu ramos inconstantes para ambos os antímeros denominados de ramos terminais da artéria basilar. O ramo emitido em seguida foi a artéria cerebelar rostral. Esta artéria apresentou-se de forma simétrica em sete $(47 \%)$ machos e três $(20 \%)$ fêmeas. A assimetria foi observada em oito $(53 \%)$ machos e $12(80 \%)$ fêmeas. Em relação ao número, a artéria cerebelar rostral se apresentou única em ambos os antímeros em 13 (87\%) machos e 10 (67\%) fêmeas, dupla no antímero direito e única no esquerdo em dois (13\%) machos e quatro $(27 \%)$ fêmeas; e dupla no antímero esquerdo e tripla no direito apenas em uma $(6 \%)$ fêmea. A artéria cerebelar rostral originou-se do ramo terminal da artéria basilar em sete (47\%) machos e seis $(40 \%)$ fêmeas e da artéria basilar em sete (47\%) machos e quatro $(27 \%)$ fêmeas. Em um $(6 \%)$ macho, a origem, no antímero direito, foi na artéria basilar e, no antímero esquerdo, no ramo terminal da basilar. Em uma (6\%) fêmea, a origem desta artéria foi, no antímero direito, na artéria carótida interna e, no antímero esquerdo, no ramo terminal da artéria basilar. Em uma (6\%) fêmea, a origem desta artéria, no antímero direito, foi na artéria basilar e no antímero esquerdo, na artéria carótida interna. Em uma $(6 \%)$ fêmea esta artéria teve dois ramos no antímero esquerdo, sendo um com origem no ramo mais caudal do ramo terminal da artéria basilar e o outro na porção mais rostral da artéria carótida interna; no antímero direito dessa fêmea, esta artéria apresentou três ramos sendo que os dois mais caudais originaram-se do ramo terminal da artéria basilar e o mais rostral originou-se da artéria carótida interna. Em uma $(6 \%)$ fêmea, no antímero direito, esta artéria apresentou dois ramos, sendo que o mais rostral originou-se na artéria carótida interna e o mais caudal no ramo terminal da artéria basilar e, no 
antímero esquerdo, apenas um ramo, que se originou do ramo terminal da artéria basilar. Na última fêmea (6\%), no antímero direito, esta artéria apresentou dois ramos, sendo que o mais rostral originou-se da artéria carótida interna e o mais caudal da artéria basilar e, no antímero esquerdo, o único ramo originou-se da artéria basilar propriamente dita.

Adiante observou-se a artéria cerebral caudal, que se apresentou simétrica em $9(60 \%)$ machos e oito $(53 \%)$ fềmeas, e assimétrica em seis (40\%) machos e sete (47\%) fêmeas. Apresentou-se única em ambos os antímeros em $12(82 \%)$ machos e 14 (94\%) fêmeas, dupla em ambos os antímeros em apenas um $(6 \%)$ macho, dupla no antímero esquerdo e única no direito em apenas um (6\%) macho e dupla no antímero direito e única no esquerdo em um $(6 \%)$ macho e uma (6\%) fêmea. Esta artéria teve origem na artéria carótida interna em $11(73 \%)$ machos e
$100 \%$ das fêmeas; e no ramo terminal artéria basilar em apenas quatro (27\%) machos. Posteriormente, observou-se a artéria cerebral média. Esta artéria foi simétrica em sete (47\%) machos e seis $(40 \%)$ fêmeas; e assimétrica em oito (53\%) machos e nove $(60 \%)$ fêmeas. Apresentou-se única em ambos antímeros em 15 (100\%) machos e 14 (94\%) fêmeas. Sua origem foi observada na artéria carótida interna, em $100 \%$ dos animais. No intervalo compreendido entre as artérias cerebral caudal e cerebral média, constatou-se a presença da artéria caróticobasilar em cinco $(33 \%)$ machos e oito $(53 \%)$ fêmeas. por último, observou-se a artéria cerebral rostral, apresentando-se única e fusionada, formando a artéria comum do corpo caloso em 100\% dos animais. Alguns destes vasos estão exemplificados na Figura 1.

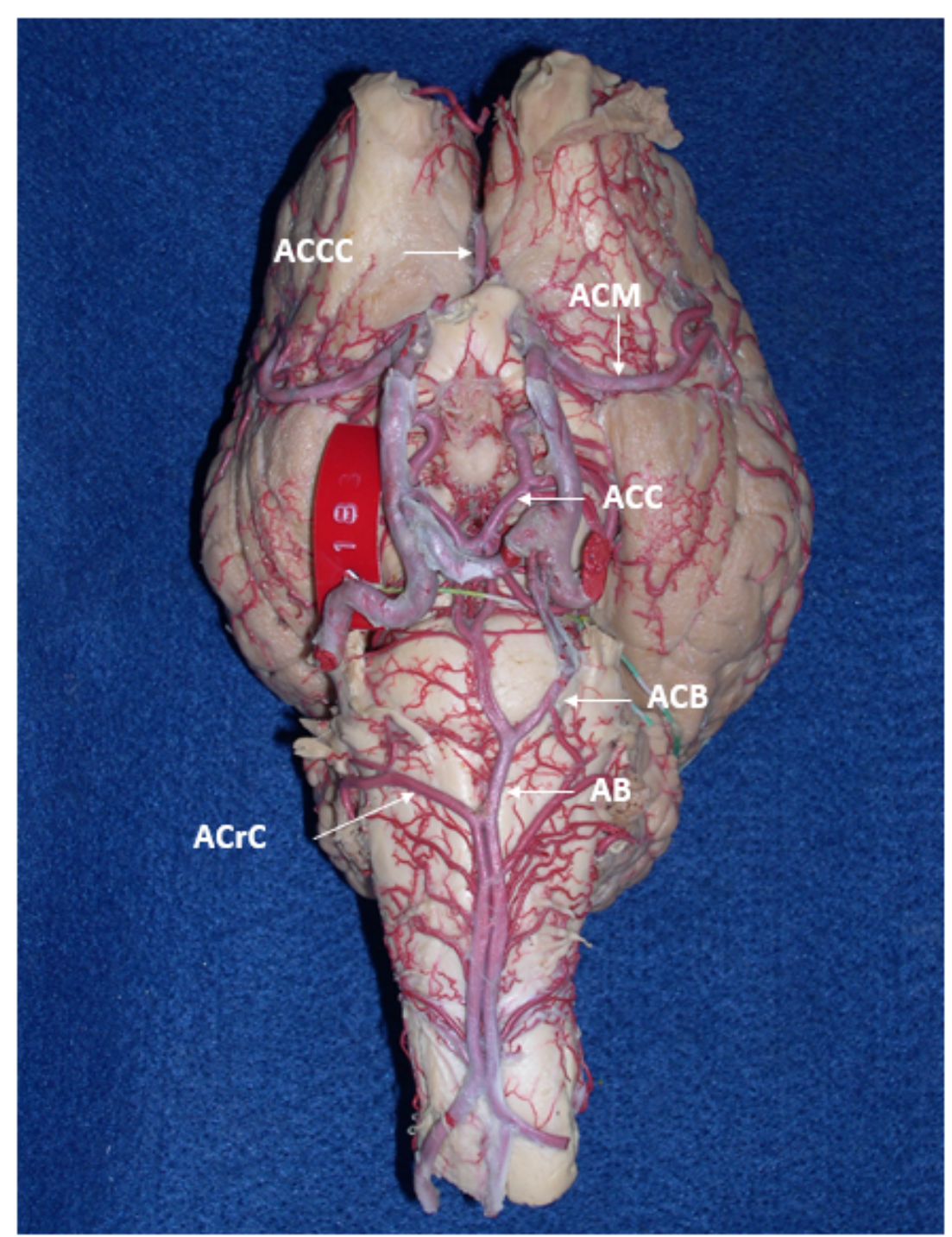

Figura 1: Fotomacrografia da vista ventral do encéfalo.

ACCC: a. Comum do corpo caloso. ACM: a. Cerebral média. ACC: a. Comunicante cauldal. ACB: a. Caróticobasilar. AB: a. Basilar. ACrC: a. Cerebelar caudal. 
O circuito arterial da base do encéfalo equino foi formado pelas artérias: comunicante caudal direita e esquerda, carótida interna em ambos os antímeros e cerebral rostral direita e esquerda. Caudalmente, apresentou-se fechado em $100 \%$ dos animais e configurado pelas artérias comunicante caudal direita e esquerda. Rostralmente, apresentouse fechado em $100 \%$ dos animais e arquitetado pelas artérias cerebral rostral direita e esquerda. Ainda rostralmente, a artéria cerebral rostral fusionou-se, formando a artéria comum do corpo caloso, onde o circuito arterial se fechou.

\section{DISCUSSÃO}

Morfometria é o estudo científico das dimensões de estruturas anatômicas macroscópicas dos indivíduos, com especial referência às suas medidas lineares e pesos. Essas medidas são muitas vezes feitas por medições diretas em amostras fixadas em solução de formaldeído, especialmente no cérebro. Os resultados variam em precisão e ainda são motivos de discussão ${ }^{14}$.

Olapade et al. ${ }^{15}$ realizaram morfometria do encéfalo de ovinos, separando-os por sexo, peso e idade, e concluíram que não houve diferença nos parâmetros do encéfalo em relação ao sexo e à idade; entretanto, animais mais pesados apresentaram aumento no comprimento do encéfalo. Na presente investigação, houve diferença entre os sexos no que se refere à largura do hemisfério esquerdo, hemisfério direito, e o comprimento do cerebelo, sendo que as fêmeas apresentaram maiores valores.

Em estudos realizados em humanos, Ribeiro et al. ${ }^{16}$ realizaram morfometria em 81 hemisférios cerebrais comparando os dois sexos e concluíram que os homens apresentaram maior comprimento e maior peso encefálico, diferente dos resultados obtidos na presente investigação em que ocorreu o contrário, sendo as fêmeas com maiores valores de largura dos hemisférios e comprimento do cerebelo em relação aos machos.

Igado e Aina ${ }^{17}$ realizaram estudos referente às medidas do cérebro de codorna (Coturnix japonica) usando machos e fêmeas e verificaram que as fêmeas apresentaram média dos valores de comprimento, peso e profundidade do cérebro superiores aos machos, parcialmente semelhante aos resultados obtidos na presente pesquisa, em que as fêmeas apresentaram maior largura dos hemisférios.

Quanto ao comprimento da artéria basilar, houve diferença nessa medida entre o sexo dos equinos estudados (Tabela 1). $\mathrm{Na}$ literatura consultada não foram observados valores correspondentes à mensuração desse vaso.
A artéria basilar, tanto nos machos quanto nas fêmeas, formou-se a partir da convergência das artérias vertebrais, direita e esquerda semelhante ao observado em gatos ${ }^{18,} 19$, em cães ${ }^{20}$ e coelhos ${ }^{21}$. Resultados diferentes foram obtidos por Jewell ${ }^{22} \mathrm{em}$ cães, pois a artéria vertebral juntava-se à artéria espinhal ventral na superfície ventral da medula espinhal, após receber anastomose das artérias occipitais originando rostralmente a artéria basilar.

De La Torre et al. ${ }^{23}$, entretanto, relataram que esta artéria em cães era formada pela união dos ramos dorsais direito e esquerdo do ramo occipitovertebral, semelhante aos resultados obtidos por Gillilan $^{24}$, que também usou o cão e o gato.

Em fetos de bovinos azebuados ${ }^{25}$ e em fetos de búfalos sem raça definida ${ }^{26}$, a artéria basilar apresentou-se como um vaso ímpar resultante da convergência dos ramos caudais, direito e esquerdo, da artéria carótida.

Ocal et al. ${ }^{27}$, em camelo (Camelus dromedrarius) e Brudnicki ${ }^{28}$, em caprinos, observaram que a artéria basilar era formada pela anastomose das artérias vertebrais e artéria espinhal ventral, distinguindo do estudo em questão em que a artéria basilar se formou a partir da fusão das artérias vertebrais direita e esquerda em todos os encéfalos dissecados.

No jacaré do papo amarelo (Caiman latirostris), Almeida e Campos ${ }^{29}$ verificaram que a artéria basilar era formada a partir da anastomose das partes convergentes do ramo caudal da artéria carótida interna, arranjo semelhante ao observado por Nazer e Campos ${ }^{30}$, no cérebro de avestruz, em que a artéria basilar apresentou-se como um vaso único em $80 \%$, de forma dupla em 13,3\% e em forma de ilha em $6,7 \%$ das amostras, diferente dos resultados da presente pesquisa no que se refere à formação da artéria basilar, porém semelhante aos resultados dos autores supra citados, pois, em equinos, a forma única foi observada em $100 \%$ dos animais.

Variações na artéria basilar foram descritas por Paiva-de-Souza et al. ${ }^{31}$, em equinos mestiços, com a frequência variável da artéria caroticobasilar, uma anastomose entre a artéria carótida interna e a artéria basilar observada na face ventral do tronco encefálico. Estes vasos também foram observados na presente pesquisa.

Depedrine e Campos ${ }^{32}$ observaram que, na raposa (Dusicyon gymnocercus), a artéria basilar originou-se da artéria vertebral direita e esquerda e pela bifurcação terminal rostral da artéria espinhal ventral; entretanto, de acordo com Wiland ${ }^{33}$, a artéria basilar na raposa forma-se através da união dos ramos da artéria vertebral e occipital. Em ambos os 
autores acima citados, os resultados obtidos diferem dos verificados na presente investigação, em que a artéria basilar formou-se exclusivamente da união das artérias vertebrais direita e esquerda.

Em roedores das espécies Hystrix cristata $^{34}$ e Spalax leucodon ${ }^{35}$, a artéria basilar originou-se da fusão das artérias vertebral direita e esquerda. Esse mesmo arranjo foi observado por Reckziegel et al. ${ }^{36}$ em estudo realizado em capivaras (Hydrochoerus hydrochoeris), por Lima et al. ${ }^{37}$ em Tamanduá Mirim, por Macedo et al. ${ }^{38}$ no Papa Mel (Eira berbara) e também nos resultados obtidos na presente investigação com equinos mestiços.

Campos et al. ${ }^{39}$ observaram que a artéria basilar, em equinos Puro Sangue Inglês, formou-se a partir da convergência das artérias occipitais direita e esquerda, diferente do que foi observado na presente pesquisa.

Em estudo realizado com encéfalos de suínos da linhagem Camborough ${ }^{22}$ por Lima et al. ${ }^{40}$, em suínos sem raça definida por Ferreira e Prada ${ }^{11}$ e no javali (Sus scrofa scrofa) por Oliveira e Campos ${ }^{41}$, foi observado que a rede admirável epidural caudal e ainda a artéria espinhal ventral foram as responsáveis pela formação da artéria basilar, diferente dos resultados obtidos com os equinos estudados, em que a artéria basilar formou-se exclusivamente da união das artérias vertebrais direita e esquerda.

Em chinchilas (Chinchilla lanigera), Araújo e Campos ${ }^{42}$ observaram que, em $96,7 \%$ dos casos, a artéria basilar formou-se pela convergência das artérias vertebral direita e esquerda, enquanto que em $3,3 \%$ foi formada pela artéria carótida interna esquerda. Os resultados desses autores, apesar de não estarem expressos separados pelo sexo, assemelhamse, em parte, com os do presente estudo, pois em $100 \%$ dos encéfalos estudados as artérias vertebrais direita e esquerda foram responsáveis pela formação da artéria basilar.

Azambuja $^{43}$ observou que, no ratão do banhado (Myocastor coypus), a artéria basilar formou-se a partir da anastomose dos ramos terminais das artérias vertebral direita e esquerda. Semelhante ao que fora observado em equinos na presente investigação e aos resultados de Ferreira e Prada $^{44}$, em estudo sobre os vasos da base do encéfalo do macaco-prego (Cebus apella).

Em todos os encéfalos analisados, a artéria basilar emitiu, como ramo principal, a artéria cerebelar caudal, que se apresentou de forma simétrica e assimétrica em relação ao antímero. A artéria basilar emitiu ramos de menor calibre para ponte e bulbo. Em seguida, emitiu as artérias cerebelares rostrais direita e esquerda. Esses resultados assemelham-se aos obtidos por Lima et al. ${ }^{18}$ e Gomes et al. ${ }^{19}$ para gatos, para Alcântara e
$\operatorname{Prada}^{45,}{ }^{46}$ para o cão e para Portugal et al. ${ }^{21}$ para coelhos.

A formação do circuito arterial do cérebro, em animais domésticos e selvagens, pode ser usado para criar modelos, com o propósito de conduzir diferentes experimentos. Sendo assim o estudo morfológico comparativo detalhado desses vasos torna-se essencial e relevante.

Estudos do circuito arterial da base do encéfalo de diferentes espécies demonstram muitas variações. Em estudos com avestruz (Struthio camelus), Nazer e Campos ${ }^{30}$ observaram que este se apresentou sempre aberto rostralmente e, em $80 \%$ dos casos, aberto caudalmente.

No jacaré (Caiman latirostris), Almeida e Campos $^{29}$ verificaram que este foi dependente exclusivamente do sistema carótico e se apresentou fechado rostralmente e caudalmente em todos os espécimes estudados, semelhante aos resultados obtidos por Azambuja ${ }^{43}$, em estudo com o Ratão do Banhado (Myocastor coypus), embora o circuito nesses animais fosse suprido exclusivamente pelo sistema vértebro-basilar. Na presente investigação, o circuito mostrou-se fechado rostralmente, em 100\% dos animais, pela presença das artérias cerebral rostral direita e esquerda, que se anastomosou, formando a artéria comum do corpo caloso, onde o circuito arterial se fechou. Caudalmente, apresentouse fechado, em $100 \%$ dos animais, sendo delimitado pelas artérias comunicante caudal direita e esquerda.

O circuito arterial do suíno (Sus scrofa domesticus) é fechado rostralmente pela presença constante da artéria comunicante rostral em 100\% dos casos e caudalmente pela presença dos ramos terminais direito e esquerdo da artéria basilar ${ }^{11}$, semelhante ao observado no javali (Sus scrofa scrofa $)^{41}$ e diferentemente dos achados na presente investigação.

No gato ${ }^{18,}{ }^{47}$, observou-se que o circuito mostrou-se fechado rostralmente pela presença da artéria comunicante rostral em $60 \%$ dos casos e caudalmente fechado pela presença dos ramos caudais das artérias carótidas do encéfalo e ramos terminais da artéria basilar em todas as amostras, distinguindo do observado na presente pesquisa, em que artérias cerebral rostral direita e esquerda são responsáveis pelo fechamento do circuito rostralmente e, caudalmente, as artérias comunicante caudal direita e esquerda.

Ocal et al. ${ }^{27}$ relataram que o circuito arterial no camelo (Camelus dromedarius) teve seu suprimento sanguíneo oriundo da artéria carótida, em cada antímero, da artéria basilar além da artéria comunicante caudal que se originou da rede admirável epidural rostral. Nos equinos deste estudo, a irrigação do circuito foi oriunda do sistema 
carótico e vértebro-basilar, também verificou-se a formação da artéria comunicante caudal, que é observada em camelos.

Araújo e Campos ${ }^{42}$, relataram que a irrigação sanguínea do encéfalo da chinchilla (Chinchilla lanígera) é formada pelo sistema vértebro-basilar, porém, em pequenas proporções, pela artéria carótida interna esquerda ou pelo sistema vértebro- basilar com contribuição da artéria carótida interna esquerda.

Reckziegel et al. ${ }^{36}$ verificaram que o circuito arterial da capivara (Hydrochoerus hydrochaeris) depende unicamente do sistema vértebro-basilar, divergindo dos resultados de Lima et $\mathrm{al}^{37}$ para o tamanduá-mirim, de Macedo et al. ${ }^{38}$ para o papa mel (Eira berbara) e dos resultados observados em equinos neste estudo, em que o suprimento sanguíneo do circuito arterial é dado pela participação do sistema carótico e vértebro-basilar.

No circuito arterial do esquilo vermelho (Sciurus vulgaris), a artéria carótida interna não contribuiu para o suprimento sanguíneo do circuito arterial, que é feito pela artéria basilar ${ }^{35}$. Arranjo morfológico semelhante foi observado por Aydin et $\mathrm{al}^{48}$, no esquilo (Spermophilus citellus) e por Aydin et al. $^{34}$, no porco-espinho (Hystrix cristata), diferentemente dos resultados deste estudo.

Lima et al. ${ }^{18}$ e Gomes et al. ${ }^{47}$ mostraram que o circuito arterial em gatos estende-se, rostralmente, desde a ponte até ao quiasma óptico, sendo representado pelas artérias carótida do encéfalo direita e esquerda, através de seus correspondentes ramos rostrais e caudais e, ainda, pelos ramos terminais em ambos os antímeros da artéria basilar em todas as preparações, diferente dos resultados da presente investigação.

Em relação à forma do circuito arterial em gatos, Lima et al. ${ }^{18}$ relataram que o arranjo dos ramos rostrais da carótida lembram uma figura elipsóide disposta transversalmente em relação à base do encéfalo, diferentemente, Gomes et al ${ }^{47}$ observaram que o circuito arterial, também em gatos, não apresentou nenhuma configuração semelhante a uma forma geométrica; entretanto, observaram predomínio da forma retilínea nos machos e cruzada nas fêmeas em relação à artéria cerebral rostral. $\mathrm{Na}$ presente investigação, o arranjo formado pelos ramos rostrais da carótida não formaram nenhum forma geométrica; alem disso, artéria cerebral rostral apresentou-se retilínea e fusionada em todos os equinos $(100 \%)$.

\section{CONCLUSÃO}

Concluiu-se que o circuito arterial do equino está na dependência do sistema carótico e vertebrobasilar, existindo um predomínio da forma retilínea da artéria cerebral rostral, e que a presença da artéria comunicante rostral não foi detectada em nenhum animal.

\section{AGRADECIMENTOS}

Os autores agradecem o apoio financeiro da FAPERJ e do CNPq.

\section{REFERÊNCIAS}

1.Pimentel LA, Oliveira DM, Galiza GJN, Rego RO, Dantas AFM, Riet-Correa F. Doenças do sistema nervoso central de equídeos no semi-árido. Pesquisa Veterinária Brasileira. 2009; 29:589-597.

2.Quiñones-Hinojosa A, Mcdermott MW, Sanai N, Ware ML. Assessment of image guided accuracy in a skull model: Comparison of frameless stereotaxy techniques vs. frame-based localization. Journal of Neurooncology.2006; 76:65-70.

3.Szeifert GT, Prasad D, Kamyrio T, Steiner M, Steiner LE. The role of the Gamma Knife in the management of cerebral astrocytomas. Progress in neurological surgery.2007; 20:150-163.

4.Kano H, Niranjan A, Khan A, Flickinger JC, Kondziolka D, Lieberman F, Lunsford LD. Does radiosurgery have a role in the management of oligodendrogliomas? Journal Neurosurgery. 2009; 110:564-571.

5.Simon M, Schramm J. Surgical management of intracranial gliomas: A review. Recent Results. Recent Results in Cancer Research.2009; 171:105-124.

6.Trah-Dinh HD, Soo YS, Jayasinghe LS. Duplication of the vértebro-basilar system. Australasian Radiology. 1991; 35:220-224.

7.De Caro R, Serafini MT, Galli S, Parenti A, Guidolin D, Munari PF. Anatomy of segmental duplication in the human basilar artery: Possible site of aneurysm formation. Clinical. Neuropathology. 1995; 14:303-309.

8.Goldstein JH, Woodcock R, Do HM, Phillips CD, Dion JE. Complete duplication or extreme fenestration of the basilar artery. American. Journal Neuroradiology, 1999; 20:149-150

9.Shroff M, Blaser S, Jay V, Chitaya TD, Armstrong D. Basilar artery duplication ssociated with pituitary duplication: A new finding. American. Journal Neuroradiology. 2003; 24:956-961.

10.Tubbs RS, Shaffer WA, Loukas M, Shoja MM, Harrigan MR, Oakes WJ. Intraluminal septation of the basilar artery: Incidence and potential clinical significance. Folia Morphologica. 2008; 67:193-195.

11.Ferreira CG, Prada ILS. O circuito arterial da base do encéfalo em suínos (Sus scrofa domesticus, Linnaeus, 
1758), formação e comportamento. Brazilian Journal of Veterinary Research and Animal Science, 2005; 42:53-60.

12.Schaller O. Nomenclatura Anatômica Veterinária Ilustrada. Manole, São Paulo, SP, 1999; p. 614. Portuguese

13.International Committee on Veterinary Gross Anatomical Nomenclature. Nomina Anatomica Veterinária. 5. ed. Hannover: Columbia, Gent, Sapporo: Editorial Committee, 2012. p.177. Disponível em http://www.wava-amav.org/Downloads/nav 2012.pdf

14.Peters M, Jancke L, Helang Y, Steinmetz H, Schlaus G, Staiger JF. Unsolved problems of comparing brain sizes in homosapiens. Brain and Cognition, 1998; 37:25485.

15.Olopade J, Obalogun BA, Oke BO, Onwuka SK. Morphometric investigation of the brain of West African dwarf sheep in Nigeria. International Journal of Morphology, 2005; 23:99-104.

16.Ribeiro PJ, Cricenti VS, Lancellotti CLP. Encefalometria na face medial do hemisfério cerebral humano: estudo em necropsias. Arquivos de Neuropsiquiatria, 2005; 63:430-436.

17.Igado OO, Aina OO. Some aspects of the neurometrics and oculometrics of the Japanese quail (Coturnix coturnix japonica) in Nigéria. Journal of Morphological Science, 2010; 27:133-135.

18.Lima EMM, Prada ILS, Carneiro E Silva FO, Severino RS, Quagliatto AL, Drummond, SS, Rodrigues GS. Estudo anatômico das artérias da base do encéfalo em gatos. Ars Veterinária, 2006; 22:1-7.

19.Gomes MS, Hernandez JMF, Alonso LS, AbiduFigueiredo M. Morfologia e ramos principais da artéria basilar em gatos. Revista Brasileira de Medicina Veterinária, 2012a; 34:206-212.

20.Pais D, Arantes M, Casal D, Casimiro M, O'neill JG. Brain stem arteries in Canis familiaris - implications in experimental procedures. Brazilian Journal of Morphological Science, 2009; 26:8-11.

21.Portugal JO, Moraes SOS, Reis RM, Gomes MS, Abidu-Figueiredo M. Morfometria do encéfalo e formação do circuito arterioso em coelhos Nova Zelândia. Biotemas, 2014; 27:147-155.

22.Jewell, P.A. The anastomoses between internal and external carotid circulation in the dog. Journal of Anatomy, 1952; 86:83-94.

23.De La Torre E, Michell OC, Netsky MA. Anatomic and angiographic studies of de vertebral basilar system in dog. American Journal of Anatomy, 1962; 110:187-198.

24.Gillilan LA. Extra and intra-cranial blood supply to brains of dog and cat. American Journal of Anatomy, 1976; 146: 237- 254.

25.Melo APF, Prada ILS. Anatomical Study of Arteries of the Base of Encephalon in Fetuses of Zebu Crossed Bovines. Brazilian Journal of Morphological Science,
$1998 ; 15: 143-149$.

26.Faria MMMD, Prada ILS. Anatomical behavior of the basilar artery of buffalo fetuses (Bubalus bubalis, Linnaeus, 1758). Revista Brasileira de Saúde e Produção Animal, 2001; 1:54-60.

27.Ocal MK, Erden H, Ogut I, Kara ME. A Quantitative Study of the Circulus Arteriosus Cerebri of the Camel (Camelus dromedarius). Anatomia Histologia Embryologia, 1999; 28: 271-272.

28.Brudnicki W. Basilar arteries of the brain in domestic goat (Capra hircus). Electronic Journal of Polish Agricultural Universities, 2000; 3:1-4.

29.Almeida L, Campos RA. Systematic study of the brain base arteries in broad-snouted caiman (Caiman latiros $\neg$ tris). Journal of Morphological Science, 2011; 28:62-68.

30.Nazer MB, Campos R. Systematization of the brain base arteries in ostrich (Struthio camelus). Journal of Morphological Science, 2011; 28:268-274.

31.Paiva-De-Souza AV, Xavier-Silva B, Antunes MS, Hernandez JMF, Scherer PO, Abidu-Figueiredo M. Frequên $\neg$ cia da artéria caroticobasilar em equinos mestiços: estudo anatômico destinado à pesquisa experimental e ao diag $\neg$ nóstico por imagem. Pesquisa Veterinária Brasileira, 2010; 30:685-688.

32.Depedrini JS, Campos R. A systematic study of the brain base arteries in the pampas fox (Dusicyon gymnocercus). Brazilian Journal of Morphological Science, 2003; 20:181-188.

33. Wiland C. The basilar artery of the brain (A. basilaris cerebri) in foxes. Folia Morphologica, 1966; 25:645-649.

34.Aydin A, Dinc G, Karan M, Ozdemir D, Yilmaz S. The morphology of circulus arteriosus cerebri in the porcupine (Hystrix cristata). Veterinarni Medicina, 2005; 50:131135.

35.Aydin A, Ilgün R, Ozkan ZE, Yilmaz S. Morphological Investigations on the Circulus Arteriosus Cerebri in MoleRats (Spalax leucodon). Anatomia Histologia Embryologia, 2008; 37:219-222.

36.Reckziegel SH, Lindemann T, Campos RA. Systematic study of the brain base arteries in capybara (Hydrochoerus hydrochaeris). Brazilian Journal of Morphological Science, 2001; 18:104-110.

37.Lima AR, Pereira LC, Branco E. Anatomia do circuito arterial do encéfalo em Tamanduá-mirim. Ciência Rural (UFSM. Impresso), 2013; 43:277-282.

38. Macedo BC, Branco E, Pereira LC, Lima AR. Estudo anatômico do circuito arterial do encéfalo em Eira berbara - relato de dois casos. Biotemas, 2014; 27: 177-183.

39.Campos A, Dos Santos D, Dos Santos Junior I, Prada IS. Artérias da base do encéfalo de equinos. Sistema occipito-basilar. Brazilian Journal of Veterinary Research and Animal Science, 2003; 40:107- 117. 
40.Lima EMM, Severino RS, Carneiro E Silva FO, Drummond SS, Bombonato PP, Campos DB, Rodrigues GS. Artérias da base do encéfalo do suíno da linhagem camborough 22. Bioscience Journal, 2005; 21:137-147.

41.Oliveira JCD, Campos R. A systematic study of brain base arteries in the wild boar (Sus scrofa scrofa). Anatomia Histologia Embryologia, 2005; 34:232-9.

42.Araújo APC, Campos RA. Systematic study of the brain base arteries and their blood supply sources in the chinchilla (Chinchilla lanigera, Molina, 1782). Brazilian Journal of Morphological Science, 2005; 22:221-232.

43.Azambuja, R.C. Sistematização das artérias da base do encéfalo e suas fontes de suprimento sanguíneo em Nutria (Myocastor coypus). 2006. 150 f. Dissertação (Mestrado em Ciências Veterinárias) - Universidade Federal do Rio Grande do Sul, Porto Alegre. 2006. http://hdl.handle.net/10183/6396

44.Ferreira JR, Prada ILS. Nomenclatura proposta para denominar as artérias da base do encéfalo do macaco- prego (Cebus apella, Linnaeus, 1766). Acta Scientiarum, 2001; 23:635- 643.

45.Alcântara MA, Prada ILS. Arteries of basis of encephalon in dogs (Canis familiaris, Linnaeus, 1758). I. Anatomical study of sources and behavior. Brazilian Journal of Veterinary Research and Animal Science, 1996a; 33:67-71.

46.Alcântara MA, Prada ILS. Arteries of basis of encephalon in dogs (Canis familiaris, Linnaeus, 1758). II. Formation and behavior of the encephalon arterial circuit. Brazilian Journal of Veterinary Research and Animal Science, v. 33, n.2, p.72-76, 1996 b.

47.Gomes MS, Dias GP, Silva MPSS, Abidu-Figueiredo M. Circulus Arteriosus Formation in Cat Brain. Eurasian Journal of Veterinary Sciences, 2012b; 28:204-208.

48.Aydin A, Ilgun S, Ozkan ZE, Yilmaz S. Morphology of the circulus arteriosus cerebri in the ground squirrel (Spermophilus citellus). Veterinarni Medicina, 2009; 54:537-542.

Protocolado em: 18 jul. 2013. Aceito em 09 Jun. 2014. 